\title{
Embodied Communication in Humans and Machines - A Research Agenda
}

\author{
IPKE WACHSMUTH ${ }^{1, *} \&$ GÜNTHER KNOBLICH ${ }^{2}$ \\ ${ }^{1}$ Center for Interdisciplinary Research (ZiF), Bielefeld University, Bielefeld, Germany \\ ${ }^{2}$ Rutgers University, Newark (NJ), USA ( *author for correspondence, e-mail: \\ ipke@techfak.uni-bielefeld.de)
}

\begin{abstract}
The challenge to develop an integrated perspective of embodiment in communication has been taken up by an international research group hosted by Bielefeld University's Center for Interdisciplinary Research (ZiF) from October, 2005 through September, 2006. An international conference was held there on 12-15 January, 2005 to define a research agenda that will explicitly address Embodied Communication in Humans and Machines.
\end{abstract}

Keywords: communication, embodiment, gesture, robots, virtual agents

\section{Introduction}

Communication, undoubtedly, is one of the most important and most complex human abilities, and it is by far not restricted to the exchange of verbal information. A significant part of the information exchanged in face-to-face interaction is expressed through other channels: body stance, gesture, facial expression, and voice quality. Communication needs an expressive body, is an embodied phenomenon throughout.

Embodiment has become one of the most promising theoretical perspectives in Cognitive Science and a challenge to AI research. Yet the role of embodiment in communication has still found comparably little attention. The challenge to develop an integrated perspective of embodiment in communication has been taken up by an international research group hosted by Bielefeld University's Center for Interdisciplinary Research (ZiF - Zentrum für interdisziplinäre Forschung) from October, 2005 through September, 2006. An international conference with highly acclaimed speakers, held there on 12-15 January, 2005 served as a first step to define a research agenda that will explicitly address Embodied Communication in Humans and Machines.

While the research group, on the one hand, aims to obtain a more profound understanding of human communication and its evolution, machine communication is another emphasis; both as a means of modeling human communicative abilities and as a means of advancing the human-technology interface. Real-time analysis of communicative signals, such as those indicating the change of speaker role (turn-taking) in dialog, seems at least as important as semantic analysis when future systems are envisioned to engage in joint action with humans. 
As a glance at the future of human-computer interaction, two virtual creatures were around at the conference. Computer scientist Kristinn Thórisson (Reykjavík University, Iceland) demonstrated Gandalf, a virtual agent guide to our galaxy. Gandalf is able to rotate and zoom projected images of planets at the user's command (uttered verbally or by gesture) and answer questions about the planets. In Bielefeld University's virtual reality laboratory, the conference participants (see Figure 1) met MAX, the Multimodal Assembly eXpert. Developed by the Bielefeld AI group, Max can imitate human gestures and exhibit humanlike synthetic speech and coverbal gesture while constructing an airplane from a construction kit in cooperation with a human partner.

In addition to humans, robots and their simulations, embodied communication in nonhuman primates will be a further research focus. The gestures observed in apes, for instance, are not only interesting phenomena in their own, but they are also indicators of elementary primate abilities to understand the perspective of conspecifics and thus lead into the quest for the origin of human communication.

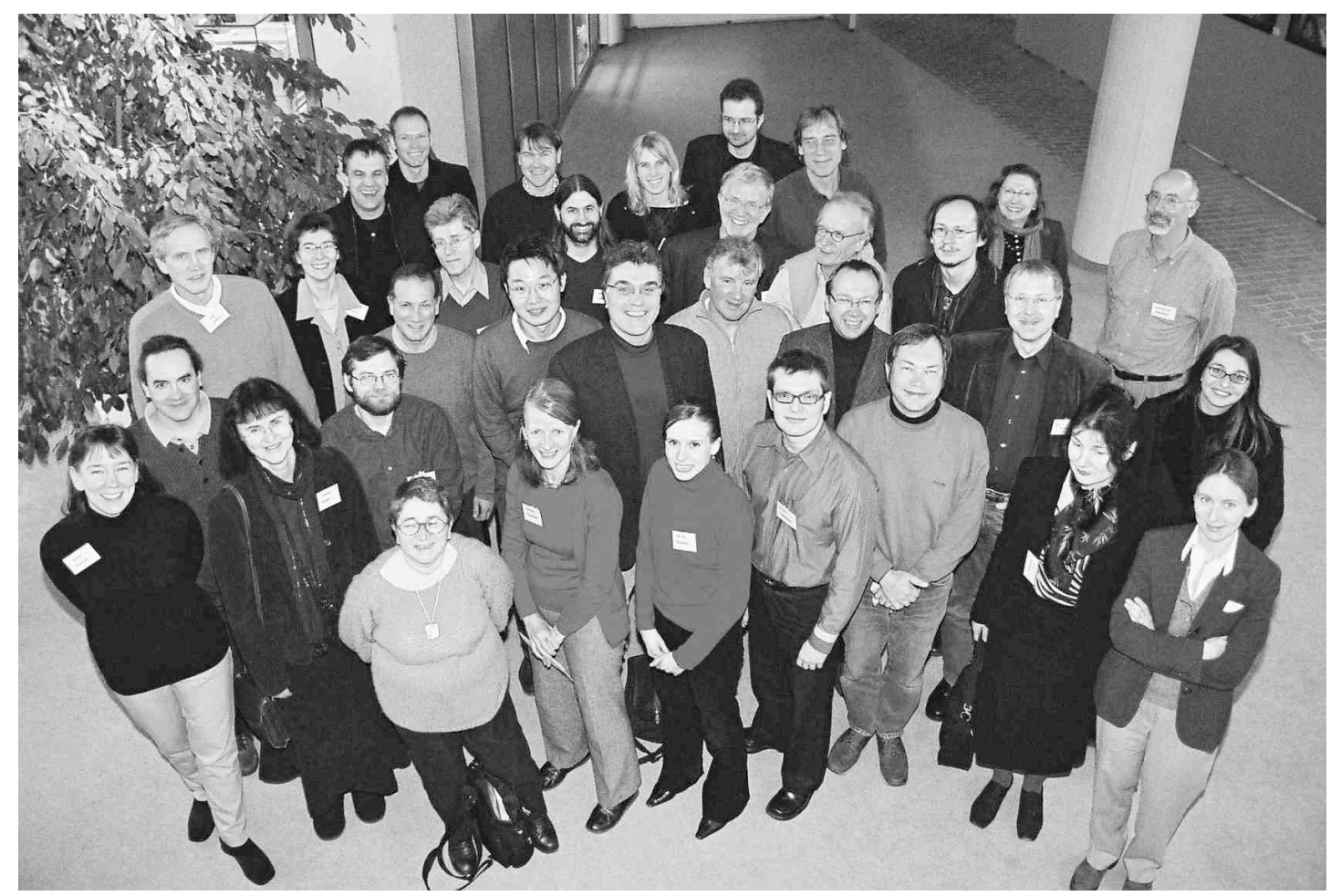

Figure 1. Participants of the Research Agenda Conference on Embodied Communication; Front row (left to right): Isabella Poggi, Catherine Pelachaud, Simone Bosbach, Kristinn Thórisson, Luc Steels, Viola Stephan, Aude Billard; Second row (left to right): Susan Duncan, Rafael Núñez, Elisabeth Ahlsén, Josep Call, Bennett Bertenthal, Sotaro Kita, Thomas Metzinger, Georg Meggle, Achim Stephan, Ipke Wachsmuth, Julie Grèzes; Third row (left to right): Jens Allwood, Manuela Lenzen, Christopher Habel, Michael Spivey, Wolfgang Prinz, Holk Cruse, Helge Ritter, Joëlle Proust, Lawrence Barsalou; Back row (left to right): Günther Knoblich, Stefan Kopp, Marcello Ghin, Natalie Sebanz, Ralf Möller, Jürgen Streeck. 


\section{Gestures and the Social Loop}

A first step on this way is the analysis of bodily communication behavior. Gestures were therefore one central theme in the conference, judged to be equally relevant for the evolution of language as for actual speaking. Whereas primatologist Josep Call (Max Planck Institute of Evolutionary Anthropology, Leipzig, Germany) reported of the rich and flexible gesture repertoire of non-human primates, psychologist Bennett Bertenthal (University of Chicago, USA), and psycholinguists Sotaro Kita (University of Bristol, UK) and Susan Duncan (University of Chicago, USA) pointed out that gestures are not simply an asset of human language but are intimately connected with speech. They are not only used to illustrate thought but they also seem to aid thinking itself. Gestures are important for the flow and the consistency of what is being said. Often they will mirror the spatial relationships between the actors in a narration. Parkinson patients, in contrast, tell poor and incoherent stories with little or no gesturing exhibited. Linguist Elisabeth Ahlsén (University of Göteborg, Sweden), however, presented cases of patients with language deficiencies who would, other than Parkinson patients, use expressive gesture to compensate for their impairment. Moreover, gestures are responsive to the gestures of others, coupling speakers and listeners in a close "social loop" in which not only their gestures but also their thoughts mutually influence each other.

\section{The Body in Human-machine Communication}

Communication, thence, is not restricted to formulating words and sentences; rather, it is an activity of the whole body. Thus embodiment should also play an important role in humanmachine interaction. The research presented by computer scientist Catherine Pelachaud (Université de Paris 8, Montreuil, France) concerns ways how to provide Embodied Conversational Agents with multimodal synchronized behavior, including gestures, adaptation to the context, and facial display. To include emotional features, Pelachaud created an Affective Presentation Markup Language (APML) which is partly based on her work with communication psychologist Isabella Poggi (Università Roma Tre, Rome, Italy). Poggi herself raised the question how the structure of the bodily communication system could be captured in rule systems. She presented ongoing research on mode-specific lexicons, such as "gestionaries," "gazeionaries," and "touchionaries," as an equivalent of dictionaries in spoken language.

\section{Bodily Roots of Interaction}

There is ample evidence that the body is also the primary and most fundamental link in social interaction. Thus, another focus of the debate was the topic of imitation and mental simulation, which was spurted by the discovery of mirror neurons responding to observing an action, a gesture, a touch, or an emotion of a conspecific. Computer scientist Aude Billard (Ecole Polytechnique Lausanne, Switzerland) described imitation as a form of embodied communication which is also of great relevance in robot learning, namely, to find a way in 
which humans and robots could learn a common means of communication. Neuroscientist Julie Grèzes (CNRS-Collège de France, Paris) demonstrated evidence that motoric brain areas of dancers are more active when they observe familiar dance movements than when observing dance of a style not familiar to them. It is only a short step from such an inner imitation to action planning. Theoretical biologist Holk Cruse (Bielefeld University, Germany) put forward the idea that the brain might generally use its model of the body to simulate future states resulting from action. In this perspective, embodied communication is grounded in a resonance between self and other that occurs on multiple levels, ranging from non-verbal actions to verbal utterances.

\section{What is a Body?}

The concept of embodiment, despite its theoretical importance, is far from clear. Does a body need flesh and blood?, linguist Jens Allwood (University of Göteborg, Sweden) asked, or might wire and metal be sufficient, or a simulation in virtual reality? The crucial part of embodiment, in his view, is grounding, a connection to the world which is absolutely required to give meaning to gestures and concepts. Psychologist Lawrence Barsalou (Emory University, Atlanta, GA, USA) presented evidence that the whole conceptual system and even abstract symbols are presumably grounded in modality-specific brain systems and that categorical knowledge may be a form of reenactment or simulation. Computer scientist Luc Steels (Free University, Brussels, Belgium and SONY Computer Science Laboratory, Paris) showed that comparably simple systems, linked to a surrounding world environment by a camera, are able not only to achieve agreement on the meaning of concepts but also to develop simple forms of syntax. His "talking heads" learn to communicate about colors and geometric shapes without being equipped with explicit rules of communication. But no single built system is like another one, he pointed out; embodiment brings about diversity and the need for a certain degree of complexity. This can make it difficult to explain the systems' behaviors - concepts like emergence and dynamic systems come into play. That the research group can hardly get by avoiding such notions became apparent in the presentation of psychologist Michael Spivey (Cornell University, Ithaca, NY, USA) on the interaction of language and perception in human communication. The mind, he emphasized, is certainly not only seated in the brain, and not even in body and brain. Rather, it seems to be situated in the triad of brain, body, and environment.

Even basic assumptions about the body that have always been taken for granted get under attack if one acknowledges recent evidence from neurological studies. When apes can learn to control a robot arm through an electrode implanted in their brains, philosopher Joëlle Proust (Institut Jean-Nicod, Paris, France) asked, then where are the borders of one's own body?

\section{Excitement and Many Questions}

A significant outcome of this conference was the spreading excitement for interdisciplinary engagement among the participants. When psychologists and roboticists linger in fascinated 
discussion of primatology videos, a first step toward fruitful cooperation is done. But only a first one: whereas computer scientist Thórisson called for a "shared code" as a precondition for a successful project, philosopher Georg Meggle (University of Leipzig, Germany) emphasized the importance of distinguishing different concepts of communication, and philosopher Thomas Metzinger (University of Mainz, Germany) pled for agreeing on a common level of description, psychologist Wolfgang Prinz (Max Planck Institute for Human Cognitive and Brain Sciences, Munich, Germany) made a case for pragmatism. There is gained little, he voiced, in the struggle over conceptual questions at the outset of interdisciplinary collaboration when it is not even clear yet which phenomena have lasting importance.

That the research group faces quite an extensive work program became obvious in the final discussion. How are bodily and verbal communication related? What is the role of the body in concept formation? What is the evolutionary relation between embodied communication and Theory of Mind? Are there specific control structures in embodied communication? How are different levels integrated in these? How can they be integrated in AI systems? What is the role of the environment? What are restrictions of embodied communication? What are the implications of such insights for prostheses and machines? By the end of the conference, a long list of challenging questions was identified which need to be answered to explore the range of the embodied communication paradigm and, hopefully, pave the way to a deeper understanding of this multifaceted phenomenon.

Details about the research group and its forthcoming events are available at the Center's Web site. ${ }^{1}$

\section{Note}

1. http://www.uni-bielefeld.de/ZIF/FG/2005Communication/

\section{Acknowledgment}

This paper is a preprint version of an article published by Springer-Verlag. The original publication is available at http://link.springer.com/article/10.1007/s10462-005-9015-5. 